\title{
Benign recurrent intrahepatic cholestasis: studies of bilirubin kinetics, bile acids, and cholangiography
}

\author{
J A SUMMERFIELD, J SCOTT, M BERMAN, C GHENT*, J R BLOOMER, \\ P D BERK†, AND SHEILA SHERLOCK
}

From the Department of Medicine, Royal Free Hospital, London, and Section on Diseases of the Liver, Digestive Diseases Branch, NIAMDD, Na tional Institutes of Health, Bethesda, Maryland, USA, and Department of Internal Medicine, School of Medicine, Yale University, New Haven, Connecticut, USA

SUMMARY Three patients with benign recurrent intrahepatic cholestasis are described. They had had between five and 16 attacks of cholestasis. Between attacks the liver function tests, including serum bile acids, were normal. Serial serum bilirubin and bile acid estimations during the cholestasis in one patient revealed a consistent discrepancy between the serum bilirubin and bile acid concentrations during three consecutive attacks. In the other two patients the serum concentrations of bile acids and"bilirubin varied in parallel. Analysis of the individual serum bile acids did not reveal high concentrations of any 'toxic' bile acid. In one patient, plasma bromsulphthalein (BSP) curves were obtained during both remission and cholestatic periods. The 45 minute retention was slightly increased $(10.8 \%)$ during remission. During the cholestasis, the 45 minute retention $(25 \%)$ and the fractional extraction coefficient $\left(\mathrm{Ke}=0.069 \mathrm{~min}^{-1}\right)$ were markedly abnormal. The hepatic clearance of unconjugated radiobilirubin was normal at all times in this patient, although during cholestasis, conjugated bilirubin refluxed from the liver to the plasma and was then cleared slowly with a half life of approximately 12 hours. Treatment with corticosteroids, cholestyramine, and phenobarbitone was unsatisfactory.

Benign recurrent cholestasis, a rare cause of cholestatic jaundice, was described by Summerskill and Walshe. ${ }^{1}$ By 1976,61 cases had been reported. ${ }^{2}$ The clinical features include the onset, usually before the age of 30 years, of recurrent attacks of cholestasis lasting several months. The cholestasis is preceded by intense pruritus, malaise, and lassitude, and, occasionally, by an erythematous rash. ${ }^{1,3}$ Diagnosis is difficult, and remains one of exclusion. The biliary tree is patent and no cause for the cholestasis, such as drugs or pregnancy, can be found. Between attacks the patients are healthy and, apart from mild portal zone fibrosis, the liver is normal. The aetiology remains obscure; the recent demonstration, how-

*Present address: Department of Medicine, University Hospital, London, Ontario, Canada.

†Present address: Department of Medicine, Mt. Sinai School of Medicine, New York, NY 10029, USA.

Address for correspondence: $\mathrm{Dr}$ J A Summerfield, Department of Medicine, Royal Free Hospital, Pond Street, London, NW3 2QG.

Received for publication 13 August 1979 ever, of very high serum bile acid concentrations at the onset of the cholestasis suggests that a bile acid transport defect may be responsible. ${ }^{4}$ Treatment is unsatisfactory, and conflicting reports surround the use of steroids, cholestyramine, and phenobarbitone. ${ }^{1,3,5,6}$ We report three further cases, including the results of repeated cholangiographic examinations, studies of radiobilirubin kinetics, serial estimations of serum bile acids, and comments on the effectiveness of various treatments.

\section{Methods}

The three male patients reported were studied at the Royal Free Hospital (London), National Institutes of Health (Bethesda, MD) and Yale University (New Haven, CT). They ranged in age from 30 to 66 years and have had between five and 16 attacks of cholestasis. Serum total bile acids in patient 1 were measured at the Royal Free Hospital by 3-hydroxysteroid dehydrogenase and fluorimetry. Gas chromatography of serum bile acids in patients 2 and 3 was performed at Yale University on 
columns of both OV 210 and SP-225Q on a Varian 1440 instrument. ${ }^{8,9}$ Bromsulphthalein clearance ${ }^{10}$ and radioactive bilirubin clearance ${ }^{11}$ studies were performed on patient 2 at the National Institutes of Health. The bilirubin studies were modified slightly in that radioactivity in plasma samples which partitioned into the polar phase of the Weber-Schalm partition system, ${ }^{12}$ representing radiolabelled conjugated bilirubin, was quantified by liquid scintillation spectrometry. In previous studies of radiobilirubin kinetics only the unconjugated bilirubin fraction was analysed. Similarly modified studies were performed in two normal volunteers and one patient with Gilbert's syndrome, who served as controls.

\section{Case histories}

\section{CASE 1 (Royal Free Hospital)}

A 51 year old white storehouseman has been jaundiced 11 times since 1943. The first episode, when he was 18 years old, began with diarrhoea, vomiting, and pruritus. One week later he became jaundiced with dark urine and pale stools. The jaundice lasted for three months with occasional sweats. When the jaundice faded he developed an

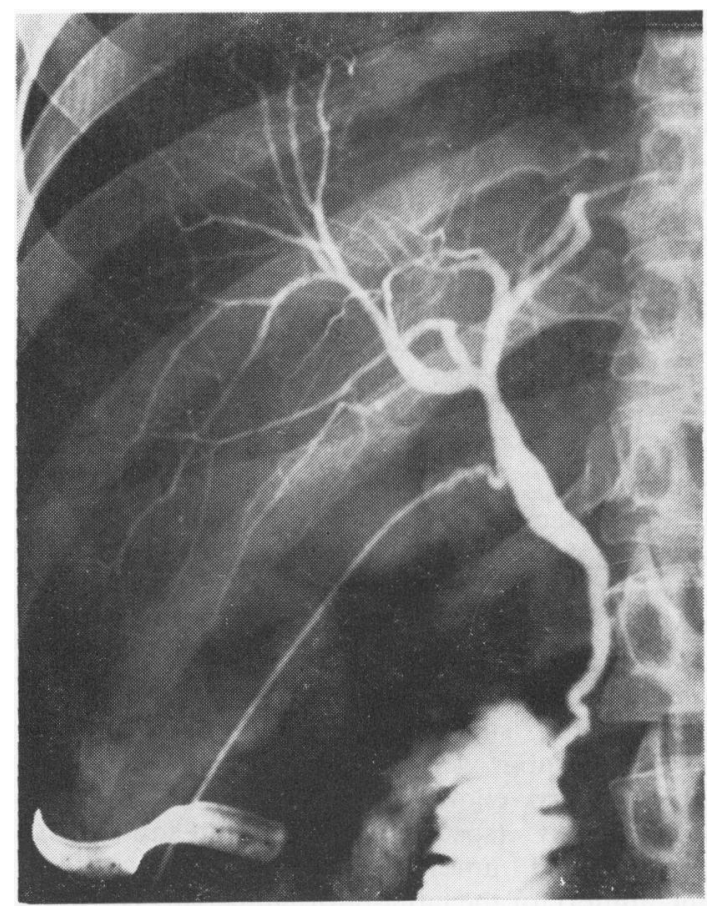

Fig. 1 Case 1. The operative cholangiogram performed seven days before a cholestatic attack shows a normal intrahepatic biliary tree and common bile duct. erythematous rash on his arms. He remained well until 1966, when, after a cold, he had a similar attack. A laparotomy revealed a normal spleen, liver, and biliary tree and a liver biopsy specimen revealed only centrilobular cholestasis. Subsequently, he had six similar episodes, often following a cold, and in 1972 had a second liver biopsy which, apart from minimal cholestasis, was normal. One episode of jaundice was treated, unsuccessfully, with steroids. In May 1975 he was referred to the Royal Free Hospital with a presumptive diagnosis of hereditary spherocytosis. Examination revealed a fit middle-aged man without hepatosplenomegaly. investigations included normal liver function tests, haemoglobin $13.4 \mathrm{~g} / \mathrm{dl}$ and reticulocyte count less than $2 \%$, but a blood film showed microspherocytosis. The diagnosis of mild hereditary spherocytosis was confirmed by osmotic fragility and autohaemolysis tests. The Coomb's tests, serum haptoglobins, and $51 \mathrm{Cr}$ red cell survival were normal. Tests for hepatitis B surface antigen (HBs Ag) by passive haemagglutination, smooth muscle, and mitochondrial antibodies were negative. An intravenous cholangiogram and endoscopic retrograde cholangiogram showed gallstones in the gallbladder but a normal biliary tree. A cholecystectomy was performed and the gallbladder contained pigment gallstones. The operative cholangiogram was normal (Fig. 1). A wedge liver biopsy showed the lobular architecture to be intact and no cholestasis was seen. The portal tracts were mildly sclerotic and infiltrated with a moderate number of mononuclear cells but no changes were seen in the bile ducts. Seven days postoperatively, he complained of itching of the palms of his hands and was noticed to be slightly jaundiced. Investigations showed, serum total bilirubin $39 \mu \mathrm{mol} / \mathrm{l}$ (conjugated $36 \mu \mathrm{mol} / \mathrm{l}$; normal: total $<17$; conjugated $<5$ ) alkaline phosphatase $21 \mathrm{KAU} / \mathrm{dl}$ (normal 3-13); $5^{\prime}$ nucleotidase $21 \mathrm{U} / 1$ (normal 1-15); glutamic oxaloacetic transaminase (SGOT) $18 \mathrm{U} / 1$ (normal 4-15); cholesterol $5.6 \mathrm{mmol} / 1$ (normal 3.0-6.5); serum total bile acid concentration $505 \mathrm{mmol} / 1$ (normal $<15)$. Treatment with cholestyramine $(16 \mathrm{~g} /$ day) did not alleviate the intense pruritus. The serum bilirubin and alkaline phosphatase concentrations slowly increased to reach a peak after two weeks. The greatest serum bilirubin was $150 \mu \mathrm{mol} / \mathrm{l}$ and alkaline phosphatase $27 \mathrm{KAU} / \mathrm{dl}$. In contrast the serum total bile acid concentration fell to $250 \mu \mathrm{mol} / 1$ during this time (Fig. 2)

During this episode of cholestasis an endoscopic retrograde cholangiogram showed a normal biliary tree and the fourth liver biopsy again showed centrilobular cholestasis without the changes of hepatitis or large bile duct obstruction. Electron microscopy 


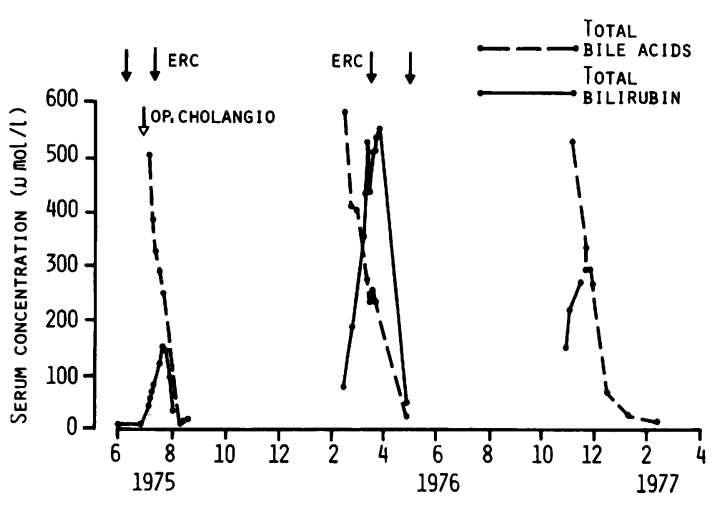

Fig. 2 Case 1. Studies of serum bilirubin ( -0$)$ and total serum bile acids ( ---$\left.)_{1}\right)$ during three consecutive cholestatic attacks. During each episode the maximum serum bile acid concentration was observed at the onset of cholestasis.

of this biopsy showed the liver cell changes associated with cholestasis and crystals in some mitochondria. Five weeks from the onset of the cholestasis, the serum total bile acid concentration had returned to the normal range ( $5 \mu \mathrm{mol} / \mathrm{l})$ (Fig. 2). The patient remained well for six months, until February 1976, when he again developed cholestasis. During the next six weeks, the serum bilirbuin rose from 77 $\mu \mathrm{mol} / 1$ to $551 \mu \mathrm{mol} / 1$ and the alkaline phosphatase from $10 \mathrm{KAU} / \mathrm{dl}$ to $60 \mathrm{KAU} / \mathrm{dl}$. The serum total bile acid concentration, however, fell from 585 $\mu \mathrm{mol} / 1$ to $210 \mu \mathrm{mol} / 1$ (Fig. 2).

\section{CASE 2 (NIH)}

This patient, a 30 year old white male, has had 16 attacks of cholestatic jaundice.

At the age of 14 years, the patient experienced the insidious onset of jaundice while he was actively engaged in school and athletics. After approximately three months of deep jaundice, pruritus, and claycoloured stools, he underwent an exploratory laparotomy. No evidence of extrahepatic obstruction was found and an operative cholangiogram was normal. After about one year, the initial attack subsided. Subsequently, he has had 15 attacks of jaundice, each lasting about two months. No precipitating events could be identified, although they often began in the Autumn. The attacks are usually heralded by fatigue, day-night reversal of sleep cycles, and paraesthesiae of the skin after a hot shower.

In addition, his appetite increased and he gained up to $13.6 \mathrm{~kg}(30 \mathrm{lb})$ in weight. A first cousin had a history of recurrent jaundice. Attacks have been treated with prednisone, phenobarbitone, cholestyramine, and recently flurazepam without noticeably influencing the course of the individual episodes. Physical examination was unremarkable apart from mild hypertension during episodes of cholestasis. There were no stigmata of chronic liver disease, abdominal tenderness, or hepatosplenomegaly. The blood count, blood urea, creatinine, serum electrolytes, cholesterol, calcium, phosphorus, uric acid, and free thyroxine have been normal. Tests for $\mathrm{HBs} \mathrm{Ag}$ and anti-HBs antibody by radioimmunoassay and passive haemagglutination, respectively, and for toxoplasmosis and infectious mononucleosis have been normal. The latex fixation test was positive but a lupus erythematosis cell preparation and antinuclear antibody have been negative. Examination by light microscopy of a liver biopsy obtained during a cholestatic episode showed cholestasis throughout the lobule, but most pronounced in the centrilobular zones. Bile plugs were present in numerous canaliculi. There were no signs of hepatitis or extrahepatic biliary obstruction. Electron microscopy revealed dilatation of the biliary canaliculi with swelling and reduction of the microvilli. Crystals and increased numbers of microbodies were found in cytoplasm of many hepatocytes and the changes associated with cholestasis were seen in the Golgi apparatus, endoplasmic reticulum, and mitochondria. With attacks of jaundice, the serum bilirubin has been as high as $680 \mu \mathrm{mol} / 1$ with alkaline phosphatase being four times normal while SGOT and SGPT were normal. During at least two episodes of cholestasis, SGOT and SGPT have been raised five to 10 -fold some 30 to 40 days after the peak of the rise in bilirubin. When he is asymptomatic, the patient's bilirubin, alkaline phosphatase, SGOT, and SGPT have been normal.

Plasma bromsulphthalein (BSP) disappearance curves were obtained during both remission and cholestatic periods, in each instance after an intravenous dose of $5 \mathrm{mg} / \mathrm{kg}$. Forty-five minute retention (normal $<5 \%$ ) was slightly increased $(10.8 \%)$ during the remission study, and markedly abnormal during cholestasis $(25 \%)$. Computer analysis of the curves $^{10}$ permitted estimation of the fractional BSP extraction coefficient, ke. Values were $0.139 \mathrm{~min}^{-1}$ and $0.069 \mathrm{~min}^{-1}$, respectively, during the two examinations (normal $=0 \cdot 124 \pm 0 \cdot 017$ (SD)). Plasma disappearance studies with unconjugated radiolabelled bilirubin were also conducted during both remission and cholestatic periods, using $1 \mu \mathrm{mol}$ bilirubin-14C (specific activity $7 \cdot 5 \mu \mathrm{Ci} / \mu \mathrm{mol}$ ) for the former and $1 \mu \mathrm{mol}$ bilirubin- ${ }^{3} \mathrm{H}$ (specific activity $25 \mu \mathrm{Ci} / \mu \mathrm{mol})$ for the latter. Normal hepatic clearance of unconjugated bilirubin was observed during both studies. ${ }^{13}$ During the cholestatic period, radioactivity leaving the plasma as non-polar unconjugated bili- 

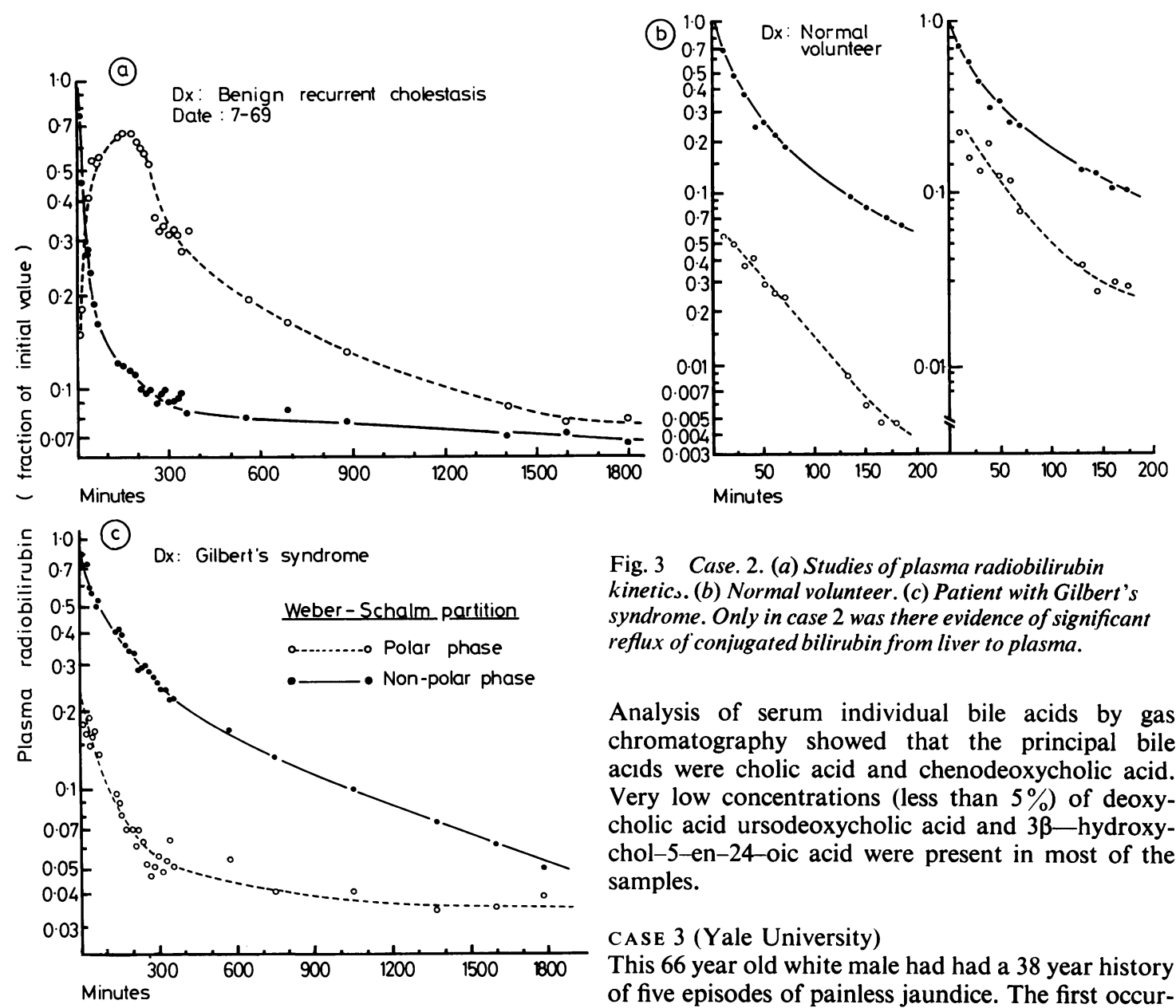

Fig. 3 Case. 2. (a) Studies of plasma radiobilirubin kinetic.. (b) Normal volunteer. (c) Patient with Gilbert's syndrome. Only in case 2 was there evidence of significant reflux of conjugated bilirubin from liver to plasma.

Analysis of serum individual bile acids by gas chromatography showed that the principal bile acids were cholic acid and chenodeoxycholic acid. Very low concentrations (less than $5 \%$ ) of deoxycholic acid ursodeoxycholic acid and $3 \beta$-hydroxychol-5-en-24-oic acid were present in most of the samples.

\section{CASE 3 (Yale University)}

This 66 year old white male had had a 38 year history of five episodes of painless jaundice. The first occurred in 1939 at the age of 28 years when the patient

rubin rapidly refluxed into the circulation as polar, conjugated bilirubin, which was then cleared slowly with a half life of approximately 12 hours (Fig. 3a). These observations, while the first of their kind in man, are similar to those reported in rats with experimental bile duct ligation. ${ }^{14}$ In contrast, no similar reflux to plasma of polar radioactivity was seen after intravenous administration of unconjugated radiobilirubin to normal volunteers or a patient with unconjugated hyperbilirubinaemia secondary to Gilbert's syndrome (Fig. 3 b, c). Serial estimations of serum total and direct bilirubin and serum fasting and post-prandial bile acids during phenobarbitone treatment $(120 \mathrm{mg} /$ day $)$ of one episode of cholestasis are shown in Fig. 4. During this treatment the serum phenobarbitone concentration remained between 60 and $129 \mu \mathrm{mol} / 1$ to achieve hepatic induction. Treatment with phenobarbitone did not appear to have any marked effect on this patient's cholestasis. developed itching followed by dark urine, pale

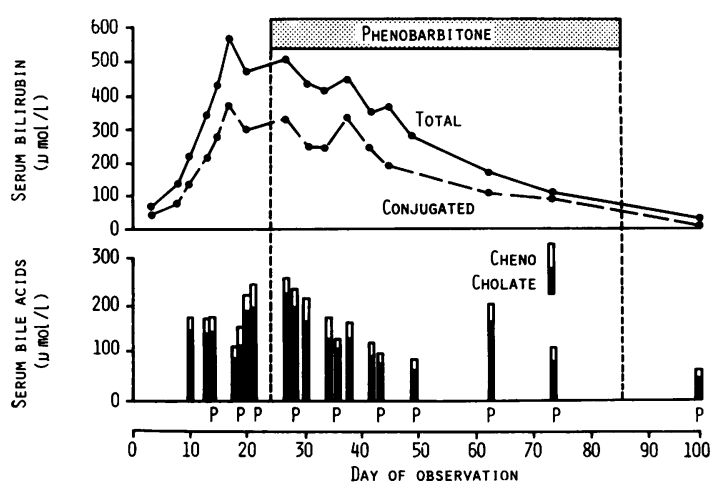

Fig. 4 Case 2. Studies of serum bilirubin and serum bile acids during a cholestatic attack treated with phenobarbitone. Cholate was the principal constituent of the serum bile acids. $(P=2 h$ postprandial estimation). 
stools, and jaundice. The episode was preceded by a severe upper respiratory infection. Jaundice, pruritus, and a $7.9 \mathrm{~kg} \mathrm{(15} \mathrm{lb)} \mathrm{weight} \mathrm{loss} \mathrm{persisted} \mathrm{for}$ three months. He was asymptomatic for the next six years, then, at the age of 34 years and again at 37 years he suffered from pruritus, dark urine, and jaundice after an upper respiratory infection. The symptoms lasted for three months. When he was 55 years old the patient had a urinary tract infection, which was treated with sulphonamides and was followed by pruritus and jaundice. At laparotomy the gallbladder contained one small stone but the operative cholangiogram was normal; the liver biopsy was consistent with intrahepatic cholestasis with Kupffer cell hyperplasia. He did well until he was 63 when jaundice, not preceded by an infection, occurred. Repeat liver biopsy showed only intrahepatic cholestasis and Kupffer cell hyperplasia. His bilirubin levels have risen as high as $425 \mu \mathrm{mol} / \mathrm{l}$, while levels of alkaline phosphatase and SGOT have, during these episodes, been normal or mildly raised. He has no family history of jaundice or liver disease and he admitted only to an occasional cocktail. On physical examination, he was healthy with no stigmata of chronic liver disease or hepatosplenomegaly, but with mild right upper quadrant pain. During one attack phenobarbitone was given (Fig. 5). Despite phenobarbitone therapy the duration of the cholestasis was similar to previous episodes. Analysis of serum individual bile acids by gas chromatography showed a similar pattern to case 2.

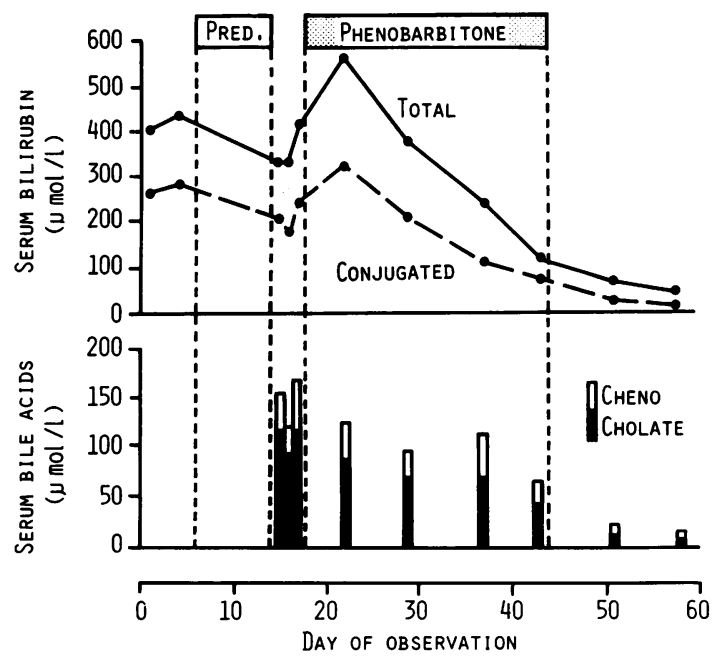

Fig. 5 Case 3. Studies of serum bilirubin and serum bile acids during a cholestatic attack treated with prednisone and phenobarbitone. Cholate was the principal constituent of the serum bile acids.

\section{Discussion}

These three patients illustrate the features of the syndrome of benign recurrent intrahepatic cholestasis (BRIC). ${ }^{1,3,15}$ The age of onset was between 14 and 28 years and the patients had suffered from five to 16 episodes of cholestasis. Each attack was usually heralded by malaise, fatigue, and intense pruritus; occasionally diarrhoea and vomiting were prominent. The prodromata were followed by jaundice, dark urine, pale stools, and usually weight loss due to the steatorrhoea. Unusually, case 2 manifested an increase in appetite, weight gain, and hypertension during attacks. The cholestatic episodes usually followed infections (cases 1 and 3) but were seasonal in case 2 . The seasonal occurrence of attacks has been noted before. ${ }^{5}$ In all our patients the cholestasis usually lasted for two to three months and they were healthy between attacks. Liver function tests during attacks reflected the cholestasis. The serum bilirubin concentration usually rose to between $170-680$ $\mu \mathrm{mol} / \mathrm{l}$. Most of the bilirubin was conjugated. The serum alkaline phosphatase was increased to about four times normal. Serum glutamic oxaloacetic transaminase (SGOT) was normal or slightly raised, but in case 2 values 10 times normal were noted.

Liver biopsies in these patients showed the changes expected in BRIC. ${ }^{1}, 3,16,17$ Biopsy specimens taken during an attack showed cholestasis throughout the lobule, but predominantly centrilobular, and bile lakes. There were no signs of hepatitis or large bile duct obstruction. The changes resolved during remissions leaving only sclerotic portal tracts infiltrated with a moderate number of mononuclear cells. Electron microscopy of the liver during an attack showed the liver cell changes of cholestasis. No specific histological changes are found in BRIC. It is noteworthy that, despite numerous attacks of cholestasis over many years, there is no permanent liver damage. ${ }^{18}$ The diagnosis of BRIC requires that the cholestasis is intrahepatic and that no other causes such as drugs, pregnancy, or haemolysis are present. In 1959, Summerskill and Walshe ${ }^{1}$ suggested the necessity of one laparotomy to exclude extrahepatic obstruction. The patency of the biliary tree can now, however, always be determined by either endoscopic retrograde cholangiography or percutaneous transhepatic cholangiography without the need for a laparotomy. Nevertheless, it is noteworthy that each of these three patients underwent laparotomy before the widespread availability of these non-surgical procedures. Operative cholangiography in the patients revealed a normal biliary tree. Furthermore, endoscopic retrograde cholangiograms performed before and during a cholestatic attack in case 1 were similar. Cholangiography 
established that the gallstones present in cases 1 and 3 were not the cause of cholestasis. Pigment gallstones in case 1 were probably related to mild hereditary spherocytosis. Their removal did not prevent subsequent attacks of cholestasis during which repeated cholangiography showed a patent biliary tree. Similarly, in case 3 , a solitary gallstone was found in the gallbladder with a normal operative cholangiogram and, despite its removal, cholestasis recurred.

The serum bile acid pattern during attacks of BRIC was studied in each of the three patients. In case 1 , a very high serum total bile acid concentration was present at the onset of each attack, when pruritus was the only complaint and jaundice was beginning to appear (Fig. 2). This discrepancy between the serum total bile acid concentration and serum bilirubin has been noted before. ${ }^{4}$ Furthermore, in case 1 , the serum total bile acid concentration fell during the attack while the serun, bilirubin rose (Fig. 2). This unexpected pattern, which was observed during three attacks, was not due to cholestyramine, which was given only once for a short period. In cases 2 and 3, however, the serum total bile acid concentration and serum bilirubin changed in parallel. We have no explanation for the difference between the patients, although one may speculate that the two patterns result from different mechanisms which may give rise to a clinically similar syndrome. In cases 2 and 3 the serum individual bile acids were estimated by gas chromatography. The principal bile acids were cholic and chenodeoxycholic acids. Very low concentrations of deoxycholic, ursodeoxycholic, and the monohydroxy bile acids, lithocholic and 3 $\beta$-hydroxychol-5-en-24-oic acids were detected. This pattern is similar to that found in any cholestasis. ${ }^{19}$ No 'toxic' bile acids were detected in sufficient concentration to account for the cholestasis. The transport defect for organic anions which develops during cholestatic periods in BRIC has been most extensively investigated for BSP. The available data have been summarised elsewhere. ${ }^{20}$ In general, plasma BSP clearance, 45 minute retention, transport maximum, and relative storage capacity are all abnormal during attacks, and normal or only slightly deranged in intercurrent periods. The findings were confirmed in case 2 . It is of interest that in two cases, abnormal BSP metabolism was detected two to three weeks before the onset of jaundice. ${ }^{5,17}$ In contrast, the studies of van Berge Henegouwen and colleagues ${ }^{21}$ have shown that, during remission, the uptake of another organic anion, indocyanine green, while normal in the familial form of BRIC, is reduced in patients with non-familial BRIC. This is further evidence for the heterogeneity of this syndrome. A few studies of plasma clearance of either non-radioactive ${ }^{3,22}$ or radioactive ${ }^{13}$ unconjugated bilirubin have shown that in BRIC, as in other predominantly cholestatic states, ${ }^{13}$ the hepatic clearance of unconjugated bilirubin is not impaired. The present study confirms a single earlier observation ${ }^{3}$ that, after the normal hepatic uptake and conjugation of bilirubin in BRIC, the conjugated pigment rapidly refluxes from the liver to plasma, rather than pursuing its normal course into the biliary system.

Conflicting claims have been made for treatments for BRIC, which may be related to the problem of assessing their efficacy in this fluctuating disease. Parenteral fat soluble vitamins (A, D, and $\mathrm{K}$ ) are obviously required during a prolonged cholestasis. Summerskill and Walshe found that corticosteroids were unhelpful, ${ }^{1}$ although a proportion of the patients may respond. ${ }^{3}$ They were of no value in cases 1 and 2 . Cholestyramine therapy has also been unrewarding, ${ }^{3}$ although Spiegal and others ${ }^{5}$ asserted that with large enough doses $(16 \mathrm{~g} /$ day) improvement occurred. However, cases 1 and 2 failed to respond to large doses of the resin. Recently, attempts have been made to reduce the serum concentrations of bile acids and bilirubin with phenobarbitone, which, among other actions, induces hepatic enzymes. Stiehl and coworkers ${ }^{6}$ reported dramatic results in one patient with BRIC. Unfortunately, phenobarbitone therapy in cases 2 and 3 had little effect on the course of the cholestasis.

In conclusion, the diagnosis of BRIC should now be reached earlier as a result of the clearer understanding of the features of the syndrome and the availability of techniques such as endoscopic retrograde cholangiography and percutaneous transhepatic cholangiography. None of these patients need now undergo laparotomy. However, the aetiology remains obscure, and, while bile acids may be in some way implicated in the pathogenesis of at least a proportion of the cases, we have found no evidence of the accumulation of 'toxic' bile acids. Treatment remains unsatisfactory, but as a proportion of patients may respond, it is worth trying brief courses of steroids, cholestyramine, and phenobarbitone.

The authors would like to thank Dr H A Khan (Plymouth, England) and Dr Lloyd Moss (Fredricksburg, Virginia) for referring cases 1 and 2 respectively; Dr. Steve Barnes and Mr Aram Chitranukroh (Royal Free Hospital) for performing the serum total bile acid estimations, Professor D N Baron (Royal Free Hospital) for biochemical data; and Professor P J Scheuer (Royal Free Hospital); and Dr Kamal Ishak (Armed Forces Institute of Pathology, Washington, DC) for examining the liver biop- 
sies and performing the electron microscopy in cases 1 and 2. The assistance of Dr R B Howe and Dr J F Martin with the studies in case 2 is gratefully acknowledged. Dr Bloomer is an investigator of the Howard Hughes Medical Institute. Dr Summerfield acknowledges the support of the Medical Research Council.

\section{References}

'Summerskill WHJ, Walshe JM. Benign recurrent intrahepatic 'obstructive' jaundice. Lancet 1959;2 686-90.

${ }^{2}$ de Prgter AGF, van Berge Henegouwen GP, ten Bokkel Huinink JA, Brandt KH. Familial benign recurrent intrahepatic cholestasis. Gastroenterology 1976;71: 202-7.

${ }^{3}$ Williams R, Cartter MA, Sherlock S, Scheuer PJ, Hill KR. Idiopathic recurrent cholestasis: a study of the functional and pathological lesions in four cases. Q J Med 1964;33:387-99.

${ }^{4}$ van Berge Henegouwen GP, Brandt $\mathrm{KH}$, de Pagter AGF. Is an acute disturbance in hepatic transport of bile acids the primary cause of cholestasis in benign recurrent intrahepatic cholestasis? Lancet 1974;1: 1249-51.

${ }^{5}$ Spiegel EL, Schubert W, Perrin E, Schiff L. Benign recurrent intrahepatic cholestasis, with response to cholestyramine. Am J Med 1965;39:682-8.

${ }^{6}$ Stiehl A, Thaler MM, Admirand WH. The effects of phenobarbital on bile salts and bilirubin in patients with intrahepatic and extrahepatic cholestasis. $N$ Engl J Med 1972;286:858-61.

${ }^{7}$ Murphy GM, Billing BH, Baron DN. A fluorimetric and enzymatic method for the estimation of serum total bile acids. J Clin Pathol 1970;23:594-8.

${ }^{8}$ Ali SS, Javitt NB Quantitative estimation of bile salts in serum. Can J Biochem 1970;48:1054-7.

${ }^{9}$ Roovers R, Evrard E, Vanderhaeghe H. An improved method for measuring human blood bile acids. Clin Chim Acta 1968; 19:449-57.
${ }^{10}$ Berk PD, Blaschke TF, Waggoner JG. Defective bromosulfophthalein clearance in patients with constitutional hepatic dysfunction (Gilbert's syndrome). Gastroenterology 1972;63:472-81.

${ }^{11 B e r k ~ P D, ~ H o w e ~ R B, ~ B l o o m e r ~ J R, ~ B e r l i n ~ N I . ~ S t u d i e s ~ o f ~}$ bilirubin kinetics in normal adults. $J$ Clin Invest 1969 ; 48:2176-90.

${ }^{12}$ Weber AP, Schalm L. Quantitative separation and determination of bilirubin and conjugated bilirubin in human serum. Clin Chim Acta 1962;7:805-10.

${ }^{13}$ Bloomer JR, Berk PD, Howe RB. Hepatic clearance of unconjugated bilirubin in cholestatic liver diseases. Am J Dig Dis 1974;19:9-14

${ }^{14} \mathrm{Ali}$ MAM, Billing BH. Plasma disappearance of conjugated and unconjugated $\mathrm{C} 14$ bilirubin in the rat with obstructive jaundice. Proc Soc Exp Biol Med 1967; 124:339-42.

${ }^{15}$ Tygstrup $\mathrm{N}$. Intermittent, possibly familial, intrahepatic cholestatic jaundice. Lancet 1960;1:1171-2.

${ }^{16}$ Biempica L, Gutstein S, Arias IM. Morphological and biochemical studies of benign recurrent cholestasis. Gastroenterology 1967;52:521-35.

${ }^{17} \mathrm{Da}$ Silva LC, De Brito T. Benign recurrent intrahepatic cholestasis in two brothers. A clinical, light, and electron microscopy study. Ann Intern Med 1966; 65:330-41.

${ }^{18}$ Schapiro $\mathrm{RH}$, Isselbacher $\mathrm{KJ}$. Benign recurrent intrahepatic cholestasis. $N$ Eng J Med 1963;268:708-11.

${ }^{19}$ Summerfield JA, Cullen J, Barnes S, Billing BH. Evidence for renal control of urinary excretion of bile acids and bile acid sulphates in the cholestatic syndrome. Clin Sci Mol Med 1977; 52:51-65.

${ }^{20}$ Beaudion M, Feldmann G, Erlinger S, Benhamou JP. Benign recurrent cholestasis. Digestion 1973;9:49-65.

${ }^{21}$ van Berge Henegouwen GP, Ferguson DR, Hofmann AF, de Pagter AGF. Familial and nonfamilial benign recurrent cholestasis distinguished by plasma disappearance of indocyanine green but not cholylglycine. Gut 1978;19:345-9.

${ }^{22}$ Brodersen R, Tygstrup N. Serum bilirubin studies in patients with intermittent intrahepatic cholestasis. Gut 1967;8:46-9. 